\title{
MECHANICAL CHARACTERIZATION OF ENERGY DISSIPATION DEVICES IN RETROFIT SOLUTION OF REINFORCED CONCRETE FRAMES COUPLED WITH SOLID WOOD PANELS
}

\author{
CAROLA TARDO $^{1 *}$, FRANCESCO BOGGIAN ${ }^{2}$, MAGNUS HATLETVEIT $^{3}$, \\ EDOARDO M. MARINO ${ }^{1}$, GIUSEPPE MARGANI ${ }^{1}$, AND ROBERTO TOMASI ${ }^{3}$ \\ ${ }^{1}$ Department of Civil Engineering and Architecture (DICAr), University of Catania \\ via S. Sofia 64, 95123 Catania, Italy \\ e-mail: carola.tardo@unict.it (*corresponding author) \\ ${ }^{2}$ Department of Civil, Environmental and Mechanical engineering, University of Trento \\ via Mesiano 77, 38123 Trento, Italy \\ ${ }^{3}$ Faculty of Science and Technology, Norwegian University of Life Sciences \\ Drøbakveien 31, 1430 Ås, Norway
}

Keywords: Seismic renovation, Reinforcement, RC frame, CLT, Steel Dissipation device

\begin{abstract}
Deep renovation interventions on existing buildings remain currently unattractive due to technical, financial and cultural/social barriers. Now that the European Union 2018 Energy Performance Directive aims to "reach the long-term greenhouse gas emission goal and decarbonize the building stock", Member States may use their long-term renovation strategies to address risks also related to fire hazards and seismic loads in addition to energy renovation. This opens a wider market for innovative approaches in retrofit of existing buildings.

The current paper illustrates the primary outcomes of an ongoing multidisciplinary Horizon 2020 research project (called e-SAFE), tackling the integration of energy, seismic and architectural renovation interventions through an innovative and combinable technological solution, applicable to non-historic RC framed buildings (i.e. built after 1950) and easily adaptable to specific climatic conditions, seismicity levels and other boundary conditions. Hence, the proposed solution will contribute to the de-carbonization of the EU building stock, reducing the occurrence of natural hazards related to climate changes, and, at the same time, to the improvement of the social resilience against earthquakes and to the enhancement of buildings architectural image.

The seismic retrofit technology consists in the external application of modular prefabricated Cross Laminated Timber (CLT) panels on the existing perimetral walls. These panels are connected to the beams of two consecutive floors by means of friction dampers and provide additional lateral stiffness and strength to the existing structure, thus reducing the storey drift demand in case of earthquake. The friction dampers cut the force transmitted by the CLT panel to the structure and dissipate energy, which further reduce the drift demand. Strength, stiffness and dissipation capacity provided by the system are controlled by modulating the thickness and the number of CLT panels, as well as the friction dampers size.

In this research phase, friction damper prototypes have been designed in order to optimize
\end{abstract}


both mechanical performance and production process. Detailed numerical models allowed to investigate the stress distribution in the dissipative connections. The results of the preliminary testing campaign will be presented and discussed in this paper, also in relation with the findings of the numerical analysis and future tests.

\section{INTRODUCTION}

A recent issue in the building renovation sector is the need of innovative and holistic design approaches, which combine both energy-efficient and anti-seismic interventions in order to meet the current Energy Performance of Buildings Directive (EPBD) [1], meanwhile improve the social resilience against earthquakes. In fact, in European seismic countries, most of the existing buildings (around 50\% of the residential stock) were built between the 1950s and 1990s, before the enforcement of the most recent energy and seismic codes [2]. Therefore, these buildings are highly energy-intensive, thus contributing to the increase in greenhouse gas (GHG) emissions and, consequently, to the climate changes and related natural hazards (e.g. floods, hurricanes, torrential rainfall, windstorms). At the same time, they are extremely vulnerable to seismic excitation, since only gravity loads were considered at the design stage, according to the old standards. Typically, these buildings are multi-storey and have a reinforced concrete (RC) frame structure with infill walls made of brittle hollow clay bricks. RC frames are often oriented along one-direction and poor structural details usually characterize members and joints, resulting in low strength, stiffness and deformation capacity of the structure in relation to lateral actions. In the event of a severe earthquake, extensive damages or collapse of these buildings are likely to environmental harm. about 160.000 deaths Combining energy reduce the seismic risk in t.
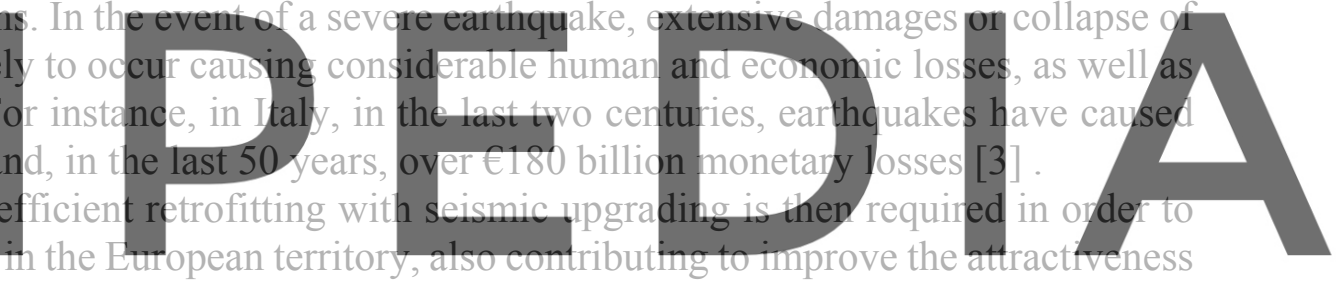

of deep renovation strategies on the existing buildings.

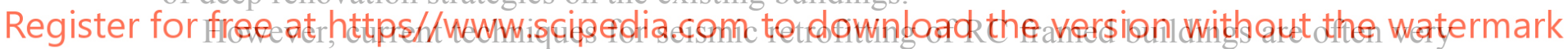
expensive, time-consuming, invasive and not reversible. Furthermore, they also are highly waste producers, resulting poorly accessible and low environmentally friendly, especially in view of a renovation that should involve most of the existing building stock. These techniques aim at increasing the strength, stiffness and deformation capacity of the structure or reducing the seismic demand. They include the jacketing of the existing structural elements with steel, RC, fibre-reinforced polymer (FRP) or textile-reinforced mortar (TRM) [4-7], the addition of new RC shear walls or steel-braced frames, located inside or outside of the existing building [8], and the installation of energy dissipation devices or base isolators [9-10].

Recently, several researches have proposed innovative integrated seismic and energy renovation solutions based on external-dry installation and on the use of low-carbon recycled or recyclable materials such as solid wood panels, e.g. Cross Laminated Timber Panels (CLT) [11]. CLT panels coupled with an insulation layer and finishing materials have been investigated in replacement of the existing masonry infill walls of RC framed buildings [12] or in addition to the external walls [13], with the main purpose of increasing the overall lateral stiffness of the structure. Special dissipative connections between CLT panels and building RC frame have also been preliminarily examined in order to provide the existing structure with 
additional seismic energy dissipation capacity [13], but more comprehensive investigations on new potential dissipative devices are required in order to optimize their structural efficiency, durability and industrial replicability.

In this framework, the paper illustrates the first outcomes of an ongoing multidisciplinary Horizon 2020 project, called e-SAFE (energy and Seismic AFfordable rEnovation solutions), aimed at investigating innovative and combinable integrated retrofitting interventions. The seismic technology (named e-CLT) that drives this intervention is based on the use of modular prefabricated CLT panels equipped with innovative energy dissipation devices.

\section{2 e-CLT SYSTEM}

The e-CLT technology consists in the external application of prefabricated CLT structural panels on the outer walls of the existing building, by connecting them to the RC beams through innovative friction dampers (Figure 1a). In occurrence of moderate ground motions, the dampers rigidly connect CLT panels to the RC structure, thus making available additional lateral stiffness and strength. Conversely, friction dampers activate in occurrence of strong ground motions, thus dissipating part of the input seismic energy (Figure 1b). These two features of the e-CLT system reduce the storey drift demand of the structure and, consequently, the damage to non-structural and structural components. Strength, stiffness and dissipation capacity provided by the proposed system are controlled by modulating the thickness and the number of CLT panels, as well as the damper size.

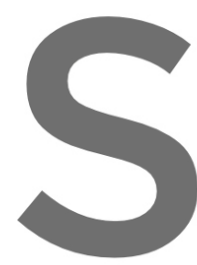
The integrated retrofittin technology with non-stru with high-performing windows that will replace structural panels integrate bio-based insulation order to improve also the endrgy performanc building [14]. Cladding solutions integrated to cover the RC beams and protect the dampers, while providing the building with architectural

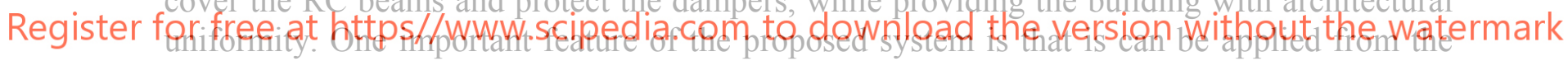
outside of the building, thus limiting the discomfort for internal users.

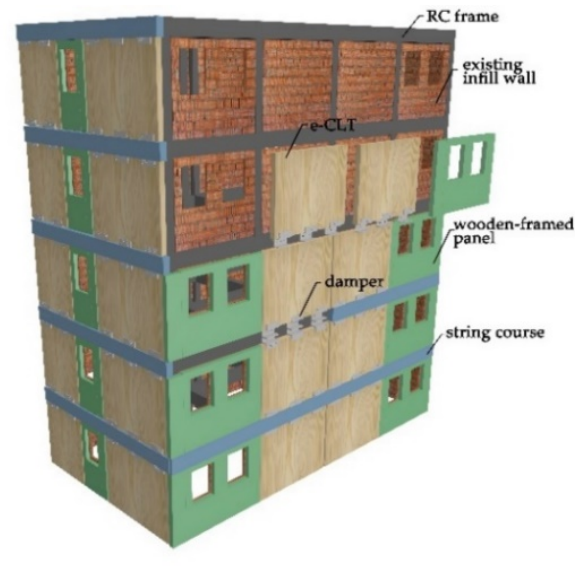

(a)

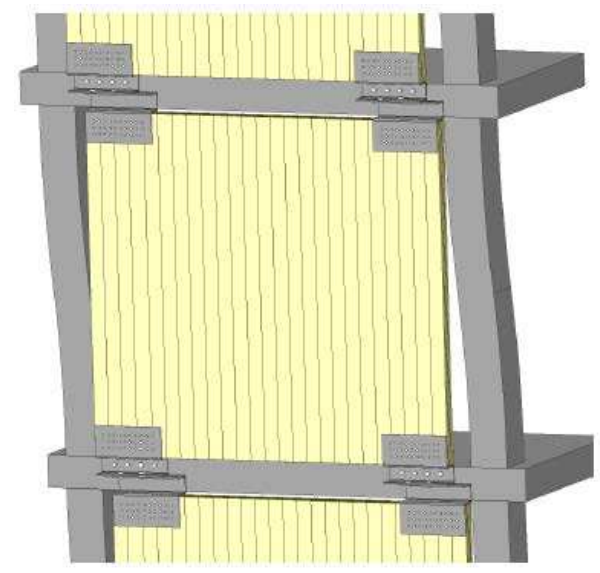

(b)

Figure 1: (a) Components of the integrated retrofitting system and (b) e-CLT technology under seismic loads 


\section{PROPOSED FRICTION DAMPERS}

Currently, the friction damper is under study with the main purpose of optimizing its shape to meet the needs of structural, technological and industrial efficiency. Specifically, four configurations of the proposed friction damper have been here investigated, in order to identify potential limits and advantages for each one (Figure 2a-d).

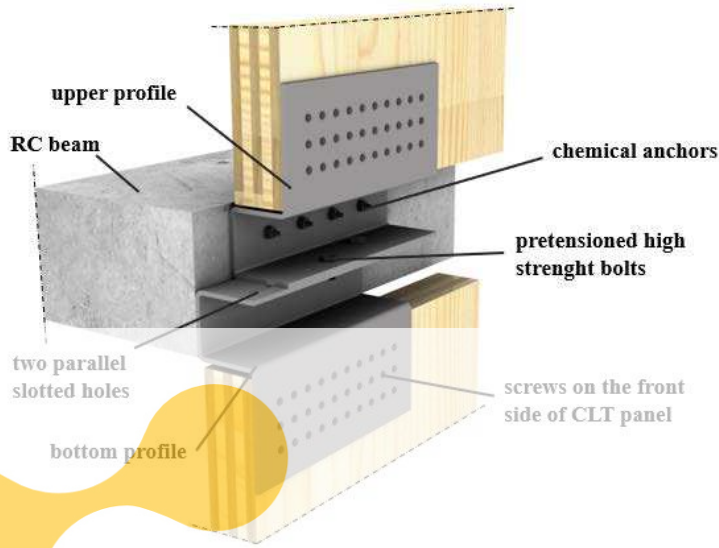

(a)

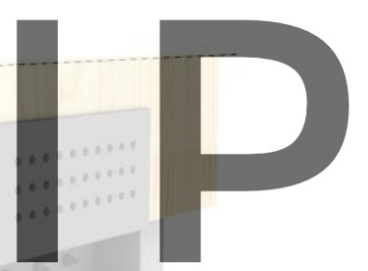

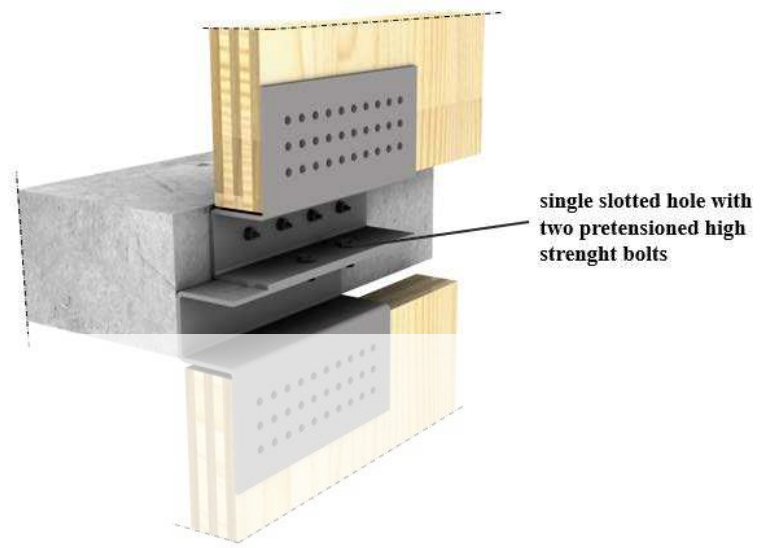

(b)
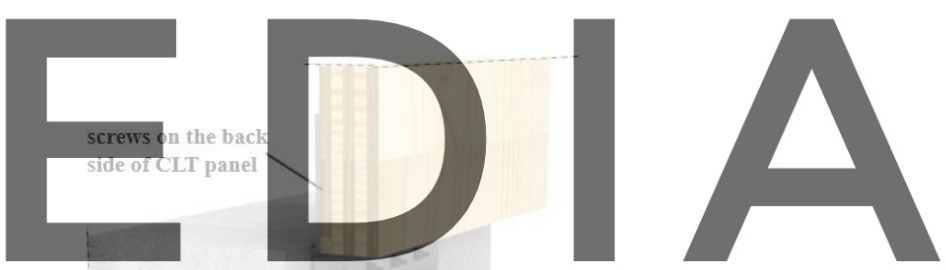

welded steel

Register for free at https//www.scipedia.com to download the version without the watermark

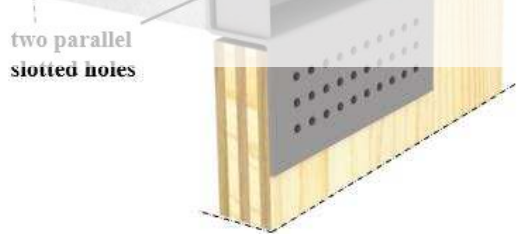

(c)

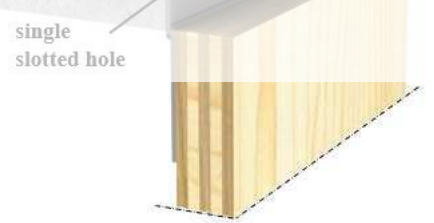

(d)

Figure 2: Friction damper configurations under study: (a) STD, (b) STD-1H, (c) STD-R; (d) ALT. (Abbreviations: $\mathrm{STD}=$ Standard; $\mathrm{R}=$ Reinforcement; $\mathrm{H}=$ Hole; $\mathrm{ALT}=$ Alternative)

In all the analysed configurations the damper consists of two cold bent steel profiles that connect the CLT panels of two consecutive storeys with the existing interposed RC beam. The upper profile is connected to the RC beam by anchor bolts. The bottom profile is provided with slotted holes and is connected to the upper one by pretensioned high strength bolts. Common timber screws connect both the upper and bottom profile to the CLT panels. The shear force is transmitted between the two profiles by means of the friction exerted in the contact surface. 
During earthquake, when the force transmitted by the damper attains the value of the friction force, the upper profile slides on the other one and dissipates seismic energy. The activation of the sliding movement between the two profiles by a predefined force allows to control the internal forces on both the damper components (steel profiles and fasteners) and CLT panels, thus properly dimensioning them and limiting or avoiding damage of these elements and the need of their replacement after the seismic event.

Specifically, in the standard configuration (damper STD in Figure 2a) the friction connection consists of two pretensioned bolts located in parallel slotted holes and the CLT/steel connections are on the front side of the CLT panels. The standard design has also been investigated in two slightly modified additional configurations: with the two pretensioned bolts located in a single centred slotted hole (damper STD-1H in Figure 2b), and with additional welded steel reinforcements at the outer edges of the bent sections of both profiles (damper STD-R in Figure 2c).
Instead, in the alternative configuration (damper ALT in Figure 2d) the CLT-steel connections are on the back side of the CLT elements, the profiles cross section is reduced and the two pretensioned bolts are in the same centred slotted hole, located closer to the vertical edge of the upper profile, in order to decrease the eccentricity of the connection. For this design, both the upper and bottom profile consists of two separate plates, welded together with a small overlap to create a gap to fit the screw heads without risking rubbing between the profiles and the RC beams. Countersunk holes in the steel plates for the screw heads have been avoided

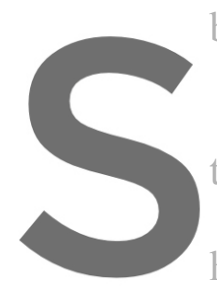
because they make industrial production more complex

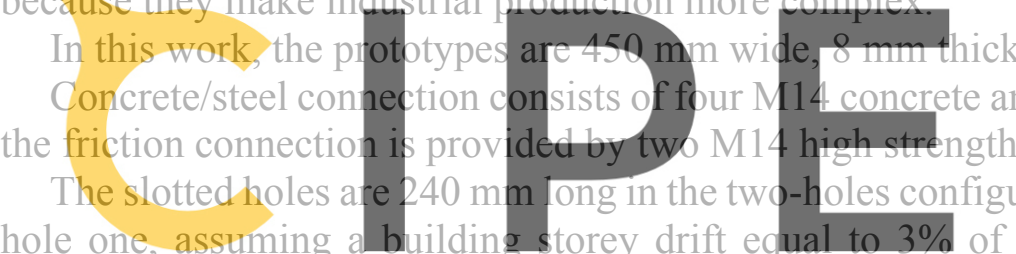

hole one, assuming a

height, i.e. $3 \mathrm{~m}$, and additional $20 \mathrm{~mm}$ tolerance in both sliding directions. Furthermore, the

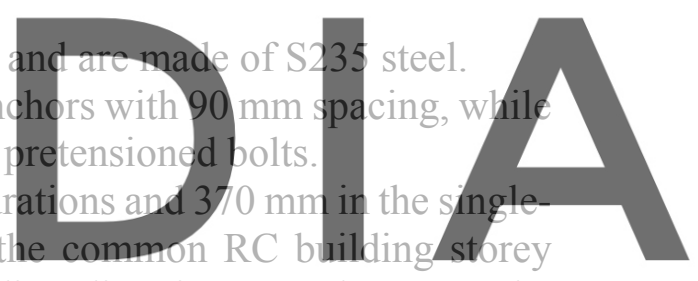

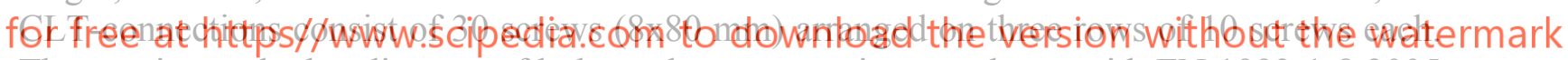

The spacing and edge distance of bolts and screws are in accordance with EN 1993-1-8:2005 and ETA-11/0030, respectively

On an industrial scale, the innovative design of the proposed friction damper enables an easy and efficient manufacturing process, since the upper and bottom steel profiles have the same development in size and are produced by cold bending and welding of steel sheets.

The damper is also designed to allow a fast and simple external installation of the proposed e-CLT technology, and to make it well adaptable to the most common RC framed buildings. For instance, in buildings with external RC flat beams and balconies, the upper profile could be directly fixed to the above overhang.

The damper configurations STD, STD-1H and STD-R allow to pre-assemble the CLT panels off-site with only the upper profiles, while the bottom profiles are fixed on site during installation in order to properly align and connect the friction surfaces of the two profiles. On the other hand, the back fitting of the CLT-steel connections in the alternative configuration ALT requires to pre-assemble the CLT panels off-site with both the profiles at the edges, and then connect them to each other and to the RC beams on site. However, in this case high precision in the damper production is required, since the adjustment of the friction surfaces alignment cannot be guaranteed on site. Another main difference between the proposed designs 
regards the inspection and replacement of the dampers after a seismic event: a swift and uncomplicated process in the first three designs, a bit more complex and time consuming in the last case.

\section{MATERIALS AND METHODS}

\subsection{Numerical modelling}

FEM analyses have been performed for each damper design in Ansys ${ }^{\circledR}$ Academic Research Mechanical, Release 19.2. Automatic meshing was used for the models, resulting in tetrahedron meshing for all damper components, except the pretensioned bolts, where hex dominant meshing was used, as shown in Figure 3a. Local mesh refinements were applied to the edge of slotted holes.

The CLT elements and RC beam have been modelled with an area equal to the adjacent steel plate one and a thickness of $100 \mathrm{~mm}$, while pretensioned bolts and screws have the same diameters as holes. A perfectly elastic material with modulus of elasticity of $210 \mathrm{GPa}$ and Poisson's ratio of 0.3 was assigned to both the profiles and pretensioned bolts. A single elastic modulus was estimated for the CLT-steel connections. Specifically, this simplified approach is achieved by assuming the characteristic axial and shear screw strain equal to the characteristic compressive CLT strain, perpendicular and parallel to the grain, respectively. The same modulus of elasticity of $200 \mathrm{GPa}$ was also used for the concrete-steel connection, as suggested from the ETAG 001. Elastic analyses have been condueted on each numerical noolel in order to preliminarily analyse the damper logding response. Damper loading consists of a Px of $30 \mathrm{kN}$, value cho vertical compressive $\phi$ one-bay RC frame with in two loading cases for each darnper design.
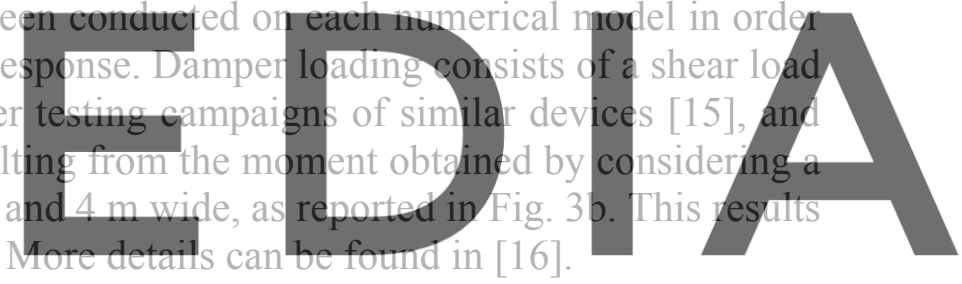

Register for free at https//wWW.scipedia.com to download the version without the watermark

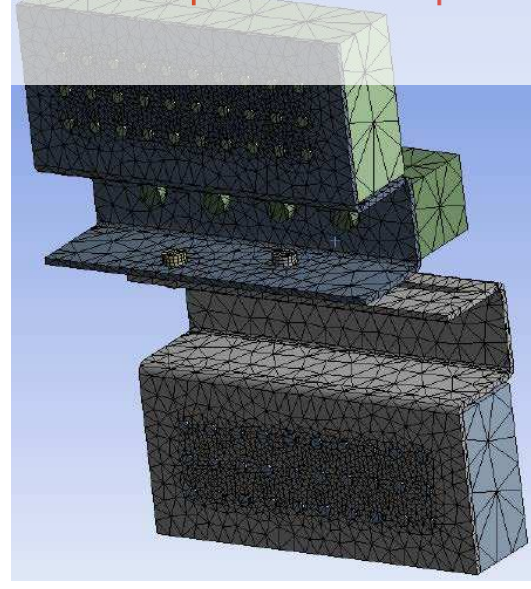

(a)

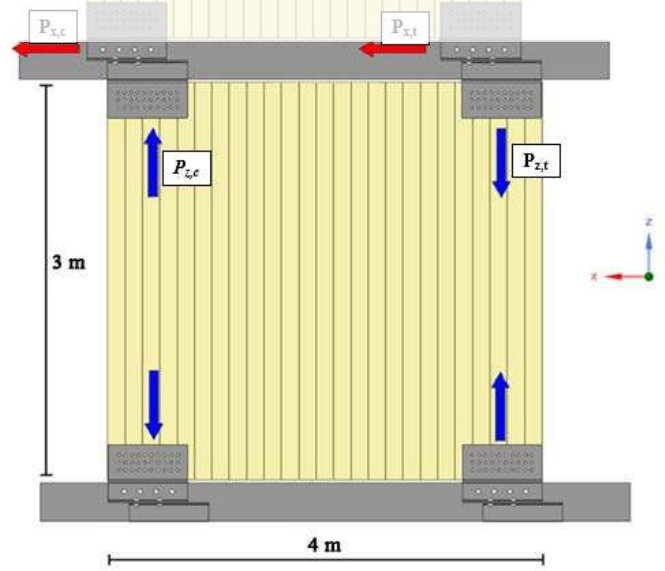

(b)

Figure 3: (a) Meshing of the STD damper model and (b) acting forces in the concept of e-CLT system (Images used courtesy of ANSYS, Inc.) 


\subsection{Test campaign}

A preliminary testing campaign is currently ongoing, with the goal being the study of the behaviour of the proposed friction connection. A specific setup was designed and built, in order to include only the dampers and no CLT elements (Figure $4 \mathrm{a}-\mathrm{b}$ ). The setup consists of a rigid steel frame, namely the columns have a $12.5 \mathrm{~mm}$ thickness and $100 \times 200 \mathrm{~mm}$ rectangular section, the bottom profile of the specimen is attached to the right column, while the top profile is in the central part of the frame and is free to slide, being connected to the actuator of the press. An additional steel cap plate and two aluminium shim layers were inserted between the profiles, in order to obtain an asymmetrical friction connection, as described by [17]. In this configuration the top profile is moved up and down by the press machine, to simulate the movement applied by the RC beam in a real building, and the bottom profile is fixed to the column, as it would be fixed to the CLT panel in reality.

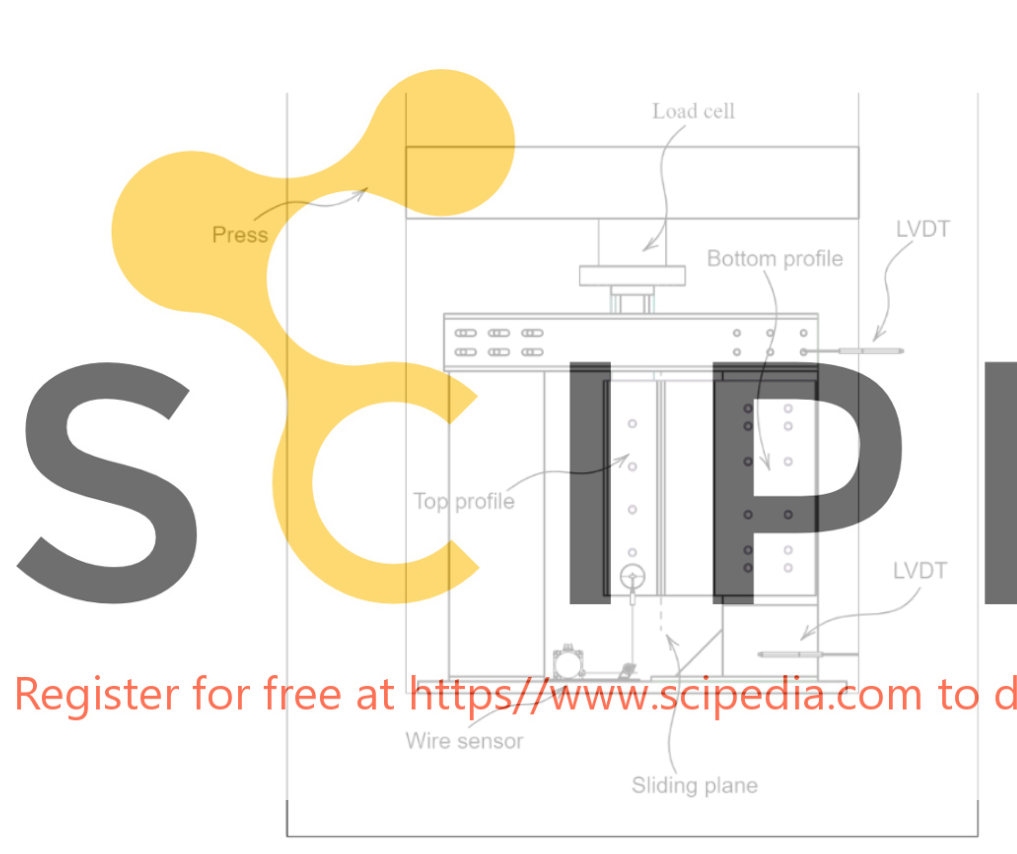

(a)

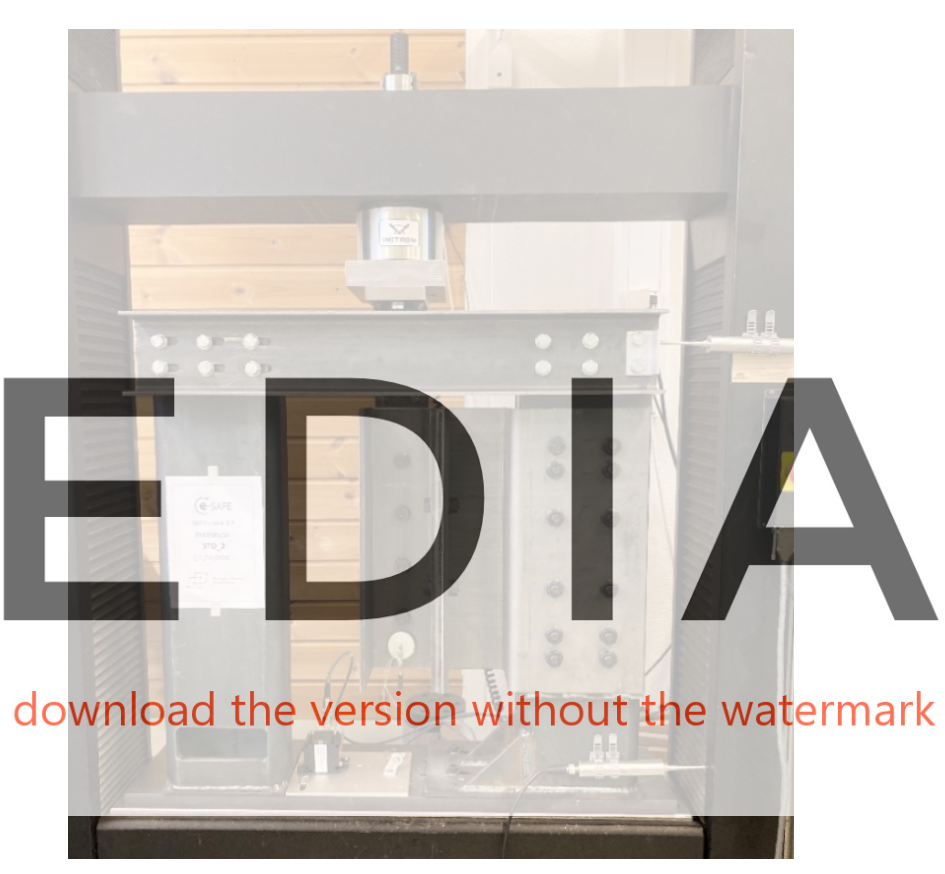

(b)

Figure 4: (a) Components of the testing setup and (b) testing setup

The damper designs considered for the test are four: the base configuration STD and STD$1 \mathrm{H}$ (with two or one slotted holes for sliding respectively), the reinforced configuration STD$\mathrm{R}$ and the alternative configuration ALT. Each specimen presents minor modifications in respect to the real configurations described in the previous sections, in order to fit the setup. Namely the bottom profile was fixed to the column with bolts, and the top profile was simplified to a C-shape, since its role in testing was only to transfer the load from the press.

The loading protocol is cyclic, adapted from ISO16670 and EN151229, which express the protocol as a function of the ultimate displacement. In this case an ultimate displacement $\mathrm{vu}=100 \mathrm{~mm}$ was assumed, estimated as $3 \%$ of the interstorey drift of a common RC frame building. The tests are conducted in displacement control. The load is measured by the load cell 
of the press, while the displacement of the top profile is recorded both by the press and by and additional wire sensor. Two horizontal LVDTs were used to acquire measures of displacement at the top and bottom of the right column, in order to verify the sufficient stiffness of the setup.

\section{RESULTS AND DISCUSSION}

\subsection{Numerical modelling}

Figures 5-7 show the results of the numerical analyses in terms of profiles stress distribution. Regarding damper STD in compression load case, the results show a maximum equivalent vonMises stress of $658 \mathrm{MPa}$ in the upper profile, specifically at the right edge of the friction connection (Figure 5a). This stress is 2.8 times the characteristic yield capacity of the S235 steel. Stress concentrations in the innermost row of screws on both the upper and bottom profiles are also observed. The second most critical stress is located at the left edge of the outer bend of the bottom profile, with a critical stress of $469 \mathrm{MPa}$. This stress is likely the result of a moment Mx.c of $1.7 \mathrm{kNm}$ resulting from the high eccentricity of the vertical force acting in the friction connection, as well as a torsion effect resulting from a large moment My,c of $4.4 \mathrm{kNm}$.

The bending and torsion effects also persist in the plate bends of damper STD in tension load case (Figure 5b). In addition, higher tension reaction force is observed in the inner pretensioned bolt compared to the outer one, resulting in a large vertical stress concentration of $687 \mathrm{MPa}$ at the vertical side of both the bends of friction connection. This could be a result of the inner bolt

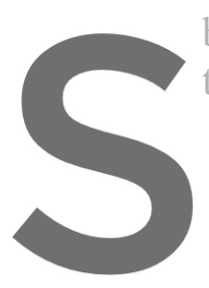
being located closer the outer bolt.

Register for free at https//www.scipedia.com to
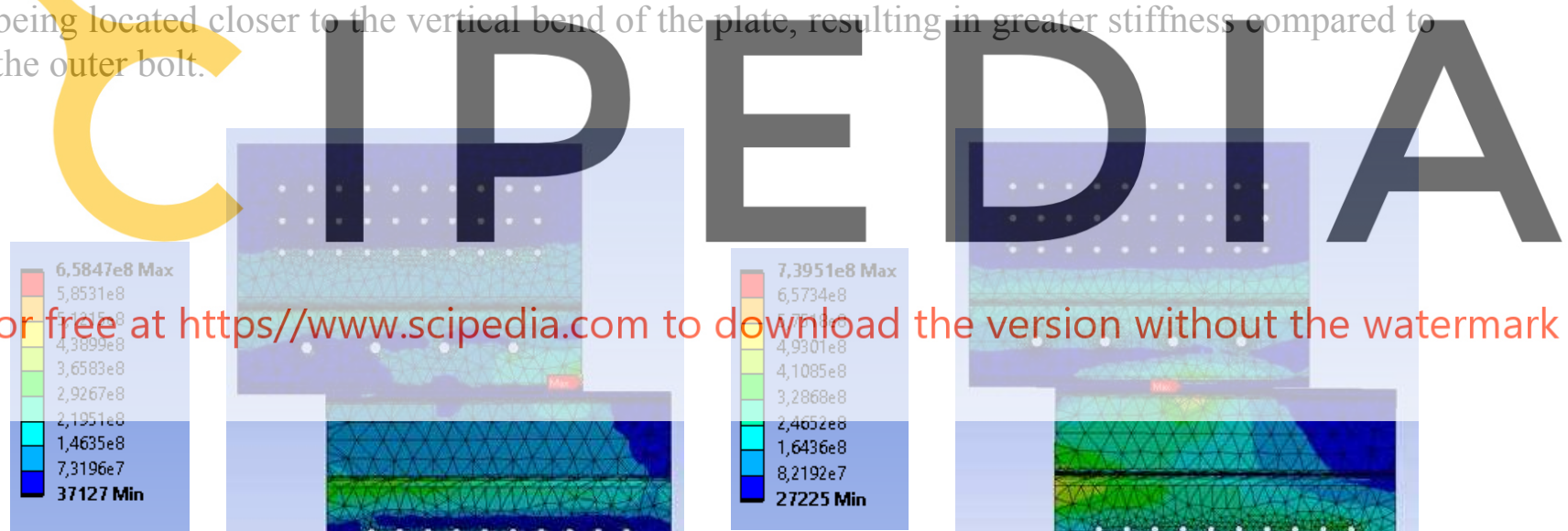

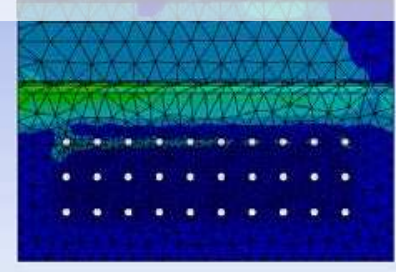

(a)

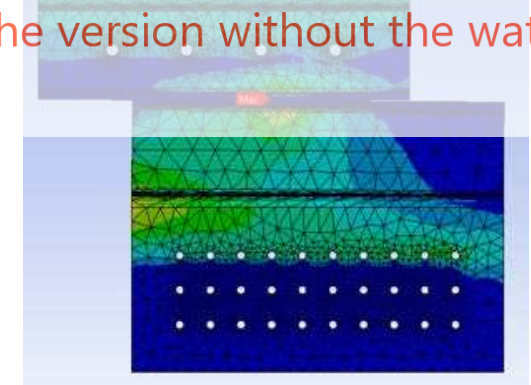

(b)

Figure 5: Equivalent von-Mises stress $\left[\mathrm{N} \mathrm{m}^{-2}\right]$ on damper STD in compression (a) and tension (b) (Images used courtesy of ANSYS, Inc.)

In damper STD-R lower stresses act on the bends of both profiles, compared to damper STD, since they are concentrated in the steel welded reinforcements, as a result of the local stiffness increase (Figure 6a). In particular, a critical stress of around $450 \mathrm{MPa}$ is located above the left reinforcement of the bottom profile in case of damper compression, while a critical stress of $628 \mathrm{MPa}$ is on the bend below the same reinforcement in tension (Figure 6b). 


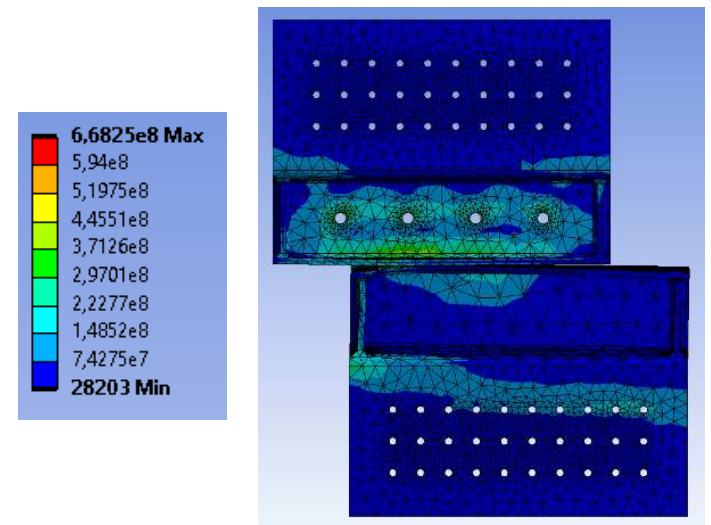

(a)

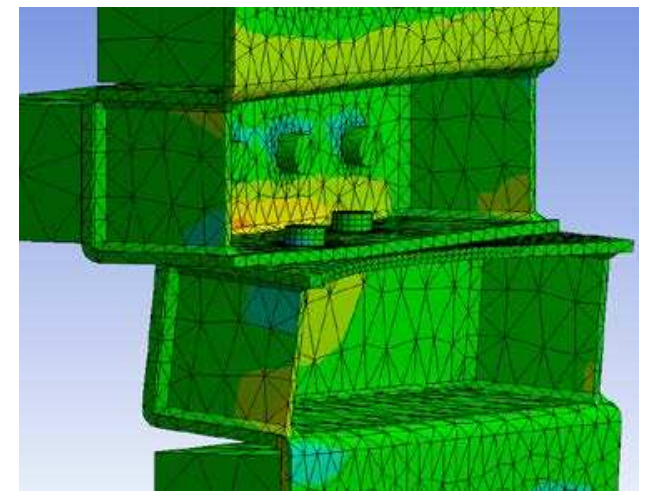

(b)

Figure 6: Equivalent von-Mises stress (a) and equivalent stress along Z-axis (b) $\left[\mathrm{N} \mathrm{m}^{-2}\right]$ on damper STD-R (Images used courtesy of ANSYS, Inc.)

The design of damper ALT is the result of the above-mentioned preliminary numerical outcomes. Lower stresses and a more even distribution are here observed, indicating a more rigid behaviour of the connection plates (Figure 7). In this configuration, the lower eccentricity of both friction and CLT-steel connections, compared to the other design, results in lower values of bending moments $\mathrm{Mx}$ and $\mathrm{Mz}$, which never exceed 0.4 and $0.7 \mathrm{kNm}$ in compression and tension, respectively. As a result, the stresses on the damper are always smaller than $150 \mathrm{MPa}$ for both load cases, except for lecal stress concentrations ardund the edges of the elongated hole, where the stress is equal to 281 and $460 \mathrm{MPa}$ for compression and tension respectively (Figure 7a-b).
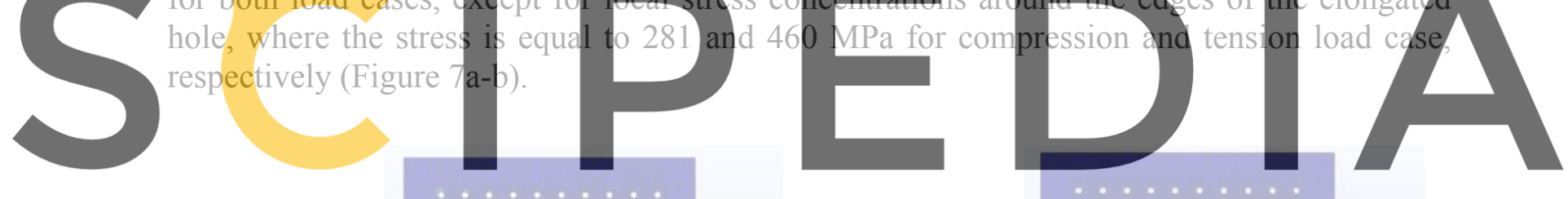

Register for free at https//www.scipedia.com to download the version without the watermark

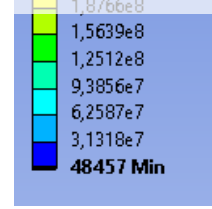

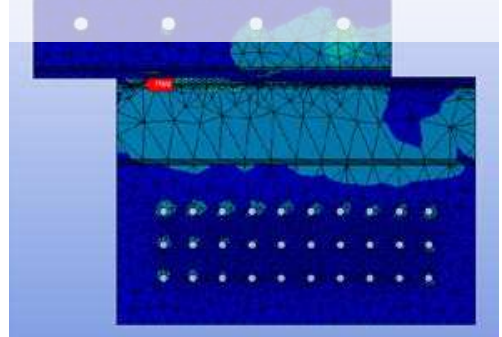

(a)

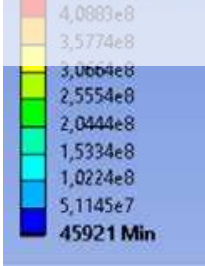

(b)

Figure 7: Equivalent von-Mises stress $\left[\mathrm{N} \mathrm{m}^{-2}\right]$ on damper ALT in compression (a) and tension (b) (Images used courtesy of ANSYS, Inc.)

\subsection{Experimental campaign}

The preliminary results of the testing campaign are presented for STD-1H and ALT damper design, since STD and STD-R suffered from big deformations, as expected from the high 
stresses found in the numerical analyses, so more investigations are required. The load protocol was applied with a speed of $0.5 \mathrm{~mm} / \mathrm{s}$, and after one initial cycle of $5 \mathrm{~mm}$ amplitude then 3 cycles for 10-20-30-40-50 mm were applied. It is important to note that this was one of the initial tests, directed at understanding the behaviour of the prototypes, and thus the input protocol was set till $50 \%$ of the ultimate displacement capacity of the specimen.

Two tests were performed on both dampers: test A with a bolt preload of $24 \mathrm{kN}$, and test B with a higher preload evaluated from the results of test A, with the goal of reaching a friction value of $30 \mathrm{kN}$. This then translated in a preload of $45 \mathrm{kN}$ and $36 \mathrm{kN}$ during test $\mathrm{B}$, for specimen STD-1H and ALT, respectively. As seen from Figure 8, the graphs of test A exhibit a behaviour that can be considered plastic, with a good stability of the hysteresis loops. Some corner chipping is present in the loops, which is common for asymmetrical friction connections, probably because of "dragging" phenomena of the cap plate not fixed to anything. Conversely, test B highlights strong differences in the behaviour of the two specimens. Damper STD-1H was subjected to big deformations in the bend of the bottom profile close to the column, which were likely the cause of big spikes in measured force and brought the test to an end before finishing all the cycles. Damper ALT exhibited better behaviour, reaching the designed 30kN value for friction. In this case though the loops present a moderate level of stability, which is worse than the ease of test A with lower preload. This is in accordance with the findings of [17], since a material with low hardness like aluminium was used as shim layer.
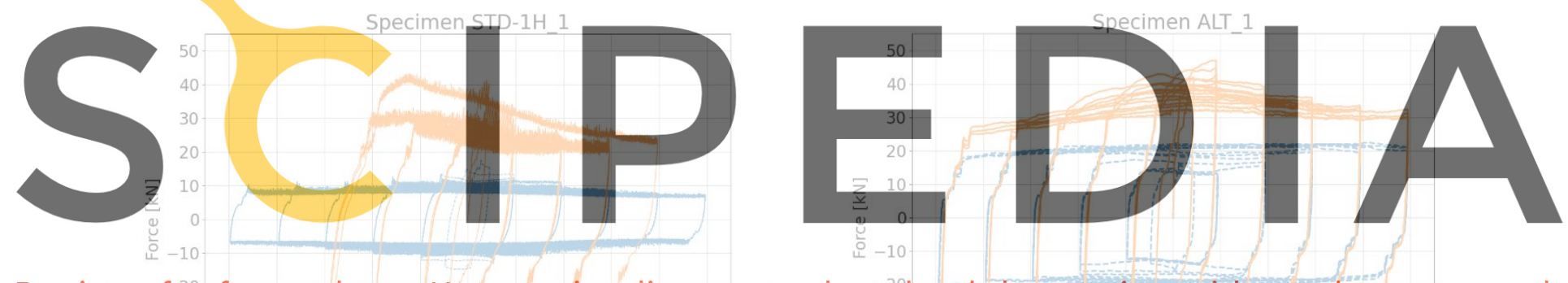

Register for free at https//www.scipedia.com to downinload the version without the watermark

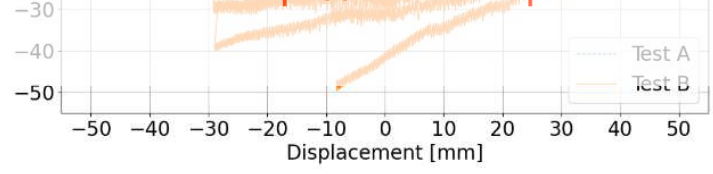

(a)

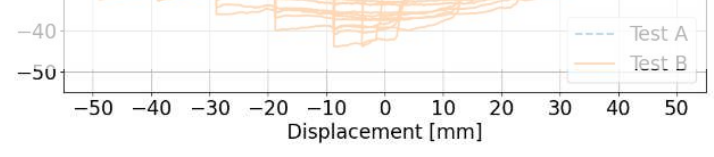

(b)

Figure 8: Force-displacement graphs for dampers (a) STD-1H and (b) ALT

\section{CONCLUSIONS AND FUTURE WORK}

This paper illustrates the first outcomes of an ongoing $\mathrm{H} 2020$ project aimed at investigating the e-CLT technology, a new seismic retrofit solution for RC framed buildings. This solution is part of an innovative and integrated renovation intervention also aimed at improving energy performance and architectural features of the building.

The e-CLT technology consists in the external application of prefabricated CLT structural panels on the existing outer walls, by connecting them to the RC beams through innovative friction dampers. Specifically, four configurations of the proposed friction damper have been 
investigated, in order to identify potential limits and advantages for each one and, consequently, to optimize both the mechanical performance and production process.

The four distinctive designs are: STD, the standard configuration; STD-1H, which has only one elongated hole instead of two; STD-R, which presents additional reinforcements in respect to the standard configuration and ALT, an alternative configuration with a different geometry and mounting procedure.

3D FEM analyses were performed in Ansys ${ }^{\circledR}$ Academic Research Mechanical, Release 19.2, which pointed out the presence of great stress concentrations in all the STD configurations, likely due to the eccentricity between the friction connection and the CLT-steel one. Design ALT was then conceived and simulated to see the effect of reduced eccentricity, and the stresses were lower than the previous cases.

A testing campaign is ongoing on the four dampers, which in part confirms what was revealed by the FEM analyses. Specimens STD and STD-R suffered major deformations, particularly in the outer bend of the bottom profile, while dampers STD-1H and ALT offered more promising results. In particular, damper STD-1H provided satisfactory hysteretic behaviour for low level of bolts preload, but experienced high deformations and loop instability at higher level of bolts preload. Specimen ALT performed well in both cases of bolts preload, with good and moderate loop stability, respectively.

New prototypes will be produced, with new geometry devised from the results illustrated in this paper and from considerations regarding the need of easy installation and maintenance of the e-CLT technology. The testing campaign will also be extended, by testing new materials as shim layers.

\section{Acknowledgements}

This project has received funding from the European Union's Horizon 2020 Innovation action under grant agreement No. 893135.

The authors would like to thank: Alberto Moretti and the Italian company Adveco s.r.l. for their relevant and continuous support during the dampers prototyping stage and for providing the dampers for the testing campaign; the master student Mathilde Marthinsen for her precious contribution during the testing campaign and the head engineer Øyvind Hansen of NMBU for his continuous support regarding the design and production of the test setup.

\section{REFERENCES}

[1] Directive 2010/31/EC. Energy performance of buildings, directive of the European parliament and of the council of 19 May 2010 on the energy performance of buildings (recast)European parliament. 2016

[2] La Greca, P.; Margani, G. Seismic and Energy Renovation Measures for Sustainable Cities: A Critical Analysis of the Italian Scenario. Sustainability 2018, 10(1), 254.

[3] Presidency of the Council of Ministers Italian Civil Protection Department. National risk assessment. Overview of the potential major disasters in Italy: seismic, volcanic, tsunami, hydro-geological/hydraulic and extreme weather, droughts and forest fire risks. 2018

[4] Chandrakar, J.; Singh, A.K. Study of Various Local and Global Seismic Retrofitting Strategies - A review. International Journal of Engineering Research \& Technology 
2017, 6(06), 824-831.

[5] Bournas, D. Innovative Materials for Seismic and Energy Retrofitting of the Existing EU Buildings; EUR 29184 EN, Publications Office of the European Union: Luxembourg, 2018.

[6] Lau, D.; Qiu, Q.; Zhou, A.; Lun, C. Long term performance and fire safety aspect of FRP composites used in building structures. Constr. Build. Mater. 2016, 126, 573-585.

[7] Tetta, Z.C.; Koutas, L.N.; Bournas, D.A. Textile-reinforced mortar (TRM) versus fiber reinforced polymers (FRP) in shear strengthening of concrete beams. Compos. Part B 2015, 77, 338-348.

[8] Rahimi, A.; Maheri, M. R. The effects of steel X-brace retrofitting of RC frames on the seismic performance of frames and their elements. Eng. Struct. 2019, 206.

[9] Barbagallo F., Bosco M., Marino E.M., Rossi P.P., Stramondo P.R. A multi-performance design method for seismic upgrading of existing RC frames by BRBs; Earthq. Eng. Struct. D. 46 (2017) 1099-1119.

[10] Mazza, F.; Pucci, D. Static vulnerability of an existing r.c. structure and seismic retrofitting by CFRP and base-isolation: A case study. Soil. Dyn. Earthq. Eng. 2016, 84, $1-12$.

[11] Bjo“rnfot A, Boggian F, Steinsvik Nyga ${ }^{\circ}$ rd A, Tomasi R (2017) Strengthening of traditional buildings with slim panels of cross-laminated timber (CLT). In: Proceedings of the 4th international conference on structural health assessment of timber structures (SHATIS'17), Istanbul, 20-22 September

[12] Stazi, F.; Serpilli, M.; Maracchini, G.; Pavone, A. An experimental and numerical study on CLT panels used as infill shear walls for RC buildings retrofit. Constr. Build. Mater. 2019, 211, 605-616.

[13] Sustersic, I.; Dujic, B. Seismic shaking table testing of a reinforced concrete frame with masonry infill strenghtened with cross laminated timber panels. World Conference on Timber Engineering, Quebec City, Canada, August 10-14, 2014.

[14] Margani, G.; Evola, G.; Tardo, C.; Marino, E.M. Energy, Seismic and Architectural Renovation of RC Framed Buildings with Prefabricated Timber Panels. Sustainability 2020, 12(12), 4845.

[15] Zheng L., Hanlin D., Xijun W., Minjuan H. Experimental and numerical investigations into seismic performance of timber-steel hybrid structure with supplemental dampers. Engineering Structures 151 (2017) 33-43

[16] Hatletveit M. Mechanical assessment of a steel dissipating system for RC buildings retrofitting with CLT panels. Master thesis, 2020. Norwegian University of Life Sciences

[17] Golondrino JC, MacRae GA, Chase JG, Rodgers GW. Behaviour of Asymmetrical Friction Connections using different shim materials. In: 2012 NZSEE Conf.; 2012. p. 1-7. 